\title{
Study of sunspot motion and flow fields associated with solar flares
}

\author{
Shuo Wang ${ }^{1}$, Chang Liu ${ }^{1}$ and Haimin Wang ${ }^{1}$ \\ ${ }^{1}$ Space Weather Research Laboratory, New Jersey Institute of Technology, \\ University Heights, Newark, NJ 07102-1982, USA \\ email: sw84@njit.edu
}

\begin{abstract}
Evolution of sunspot structure and photospheric magnetic fields are important to understand how the flare energy is built up and released. With high-resolution optical data, it is possible to examine in details the optical flows of the photosphere and their relationship to the flaring process. Using G-band and Stokes-V data obtained with Hinode Solar Optical Telescope (SOT), we study the sunspot motion and flow fields associated with the 2006 December 13 X3.4 flare in NOAA AR 10930. We calculate the centroids of the delta spot umbrae lying in opposite magnetic polarities, and use two different methods to derive the photospheric flow fields of the AR. We find that the shearing motion before the flare changes to unshearing motion associated with the eruption. A decrease of average velocity of shear flow is found to be associated with the flare, with a magnitude of $0.2 \mathrm{~km} \mathrm{~s}^{-1}$.

As a related study, we also test implementing the recently developed differential affine velocity estimator for vector magnetograms (DAVE4VM; Schuck, P. W 2008) technique for the magnetic field observations obtained by the Big Bear Solar Observatory (BBSO) and Helioseismic Magnetic Imager (HMI) on board the Solar Dynamic Observatory (SDO). Using this method to analyze changes of active region magnetic fields associated with flares may shed new light on the cause and effect of flaring process.
\end{abstract}

Keywords. Sun: magnetic fields, sun: flares, sunspots

\section{Introduction}

Evolution of sunspot structure and photospheric magnetic field associated with solar flares have recently drawn increasing attention. A sudden change of center-of-mass (CoM) separation of the two opposite polarities of a $\delta$ spot structure was found to be associated with large flares (Wang 2006). In the direction parallel to the magnetic polarity inversion line (PIL), the CoM separation always decreases, while in the direction perpendicular to the PIL, the CoM separation increases and decreases when the active region magnetic fields have a divergence and convergence motions, respectively. To further understand the variation of $\mathrm{CoM}$ separation of sunspots related to the flaring process, we investigated the high-resolution Hinode observations (Kosugi et al. 2007) to obtain the spot motion and flow fields associated with the 2006 December 13 X3.4 flare. Such a study can be greatly advanced with the aid of temporal evolution of the three-dimensional (3D) velocity fields of the source active region, which can now be achieved by the DAVE4VM technique using vector magnetic field observations.

The DAVE4VM models motion of a vector of images with normal component of the ideal magnetic induction equation: $\partial_{t} B_{z}+\nabla_{h} \cdot\left(B_{z} V_{h}-V_{z} B_{h}\right)=0$, where the plasma velocity $V$ and the magnetic fields $B$ are decomposed into a local Cartesian coordinate system with vertical direction along the z-axis and the horizontal plane, denoted generically by the subscript $h$, containing the $\mathrm{x}$ - and y-axes. Here we applied this method to 

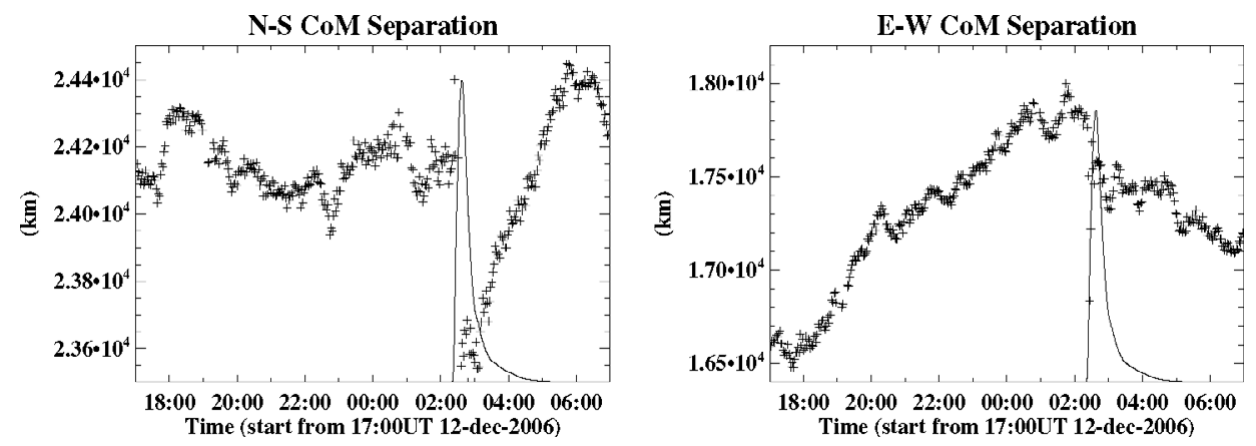

Figure 1. Time profile of CoM separation (pluses) in G-band intensity of NOAA AR 10930 between the northern (positive) and southern (negative) umbrae in the north-south (left) and east-west (right) direction, overplotted with GOES 1-8 A soft X-ray light curve.
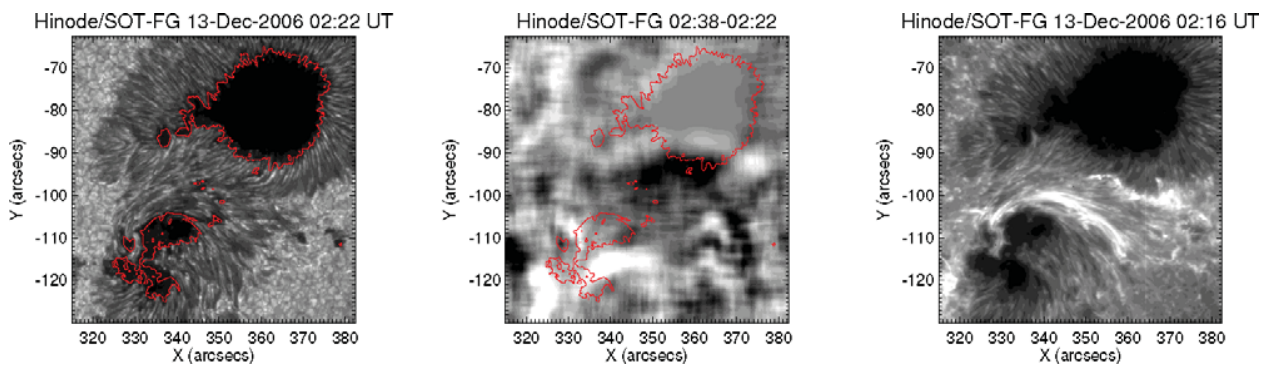

Figure 2. From left to right: Pre-flare G-band image, G-band difference image between postand pre-flare states, and a Ca II $\mathrm{H}$ image of the flare. Red contour shows position of umbra boundary.

NOAA AR 10365 on 2003 May 28 and NOAA AR 11057 on 2010 March 29, the latter of which belongs to the new solar cycle 24 .

\section{Data Sets and analysis methods}

On 2006 December 13, an X3.4 two-ribbon flare occurred in NOAA AR 10930. Hinode fully covered this event, and we used G-band (430.5 nm) and Stokes-V (Fe I $630.2 \mathrm{~nm}$ ) images obtained by its onboard SOT (Tsuneta et al. 2008). Active region flow fields from before to after the flare were derived and compared using both the DAVE (Schuck, P. W 2006) and Fourier LCT (Welsch et al. 2004) techniques.

Vector magnetograms of NOAA AR 10365 associated with an X3.6 flare on 2003 May 28 were taken by BBSO. For NOAA AR 11057 on 2010 March 29, magnetic field observation was made by the state-of-the-art SDO/HMI. The 3D active region velocity fields of both active regions were derived using DAVE4VM. The initial test shows the promise that we will be able to carry out systematic studies of flare-related temporal evolution of photospheric flow field in the near future.

\section{Results}

Figure 1 shows that the shearing motion of the two sunspots with opposite polarities of the $\delta$ configuration of NOAA AR 10930 changes to unshearing motion in the direction parallel to the PIL (east-west), which seems to be cotemporal with the GOES-class X3.4 flare. In Figure 2, the difference images in G-band intensity are taken immediately 

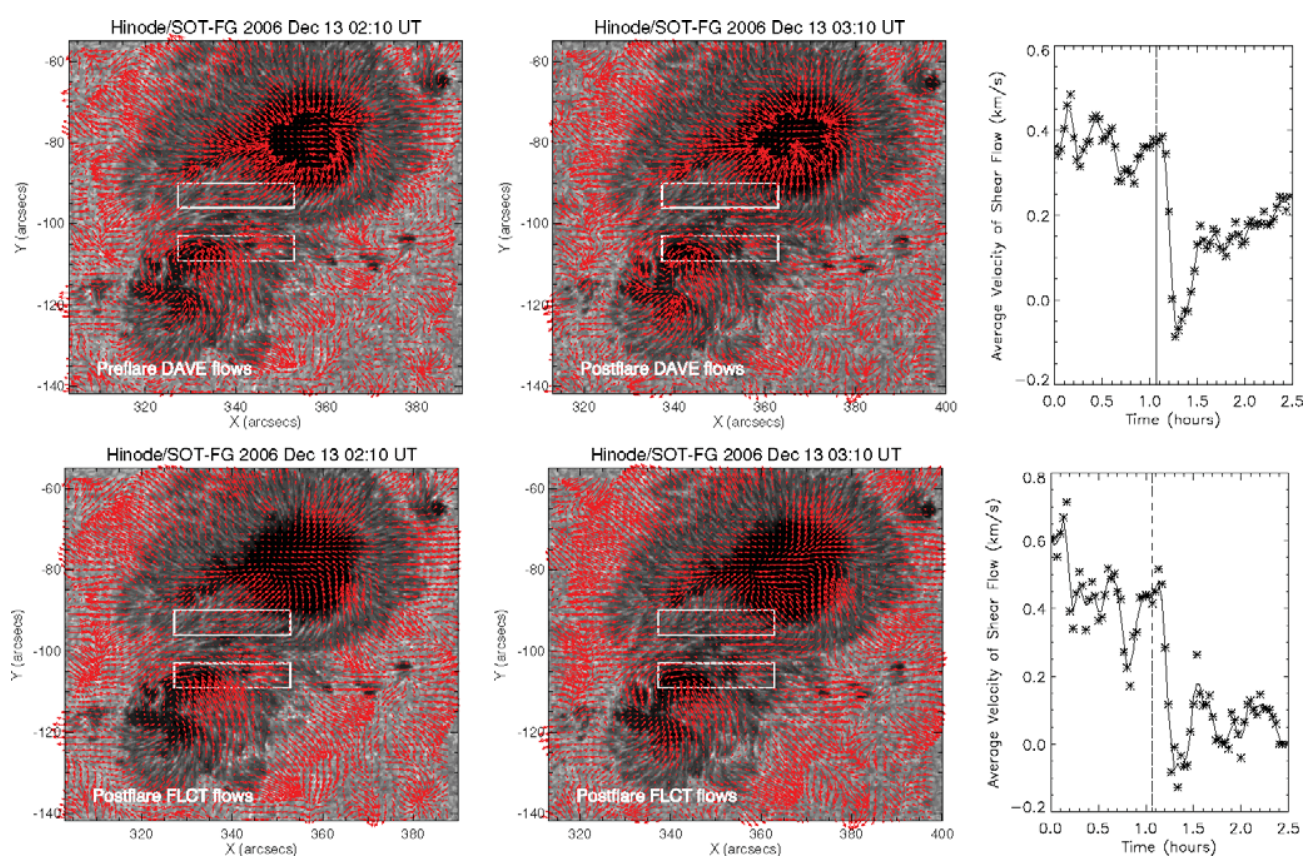

Figure 3. Flow fields for the pre-flare (first column) and post-flare (second column) states derived using the DAVE (upper panels) and FLCT (lower panels) methods. The third column shows the time profile of shear flows calculated as the difference of flows within the two boxed regions, and the start time is 1:10:40 UT 2006 December 13.

before and after the flare (post-flare 02:38:36 UT minus pre-flare 02:22:35 UT state), which reveals the enhancement (black) and decay (white) of sunspot structure that mainly occurred at penumbral regions. The enhancement and decay patterns appear to be consistent with the sudden change in the CoM separation. A Ca II image at 02:16 UT shows the position of initial flare ribbons. The locations of penumbral decay and enhancement are related to flare emissions similar to previous studies (e.g., Liu et al. 2005). In Figure 3, the white boxes denote the regions where the temporal evolution of the mean shear flow (curves with asterisks) is calculated. Overall, the DAVE method seems to produce more consistent results of flow field with the sunspot morphology. The decrease of average velocity of shear flow around the flaring PIL with a magnitude of $0.2 \mathrm{~km} \mathrm{~s}^{-1}$ is comparable to that obtained by Tan et al. 2009 using LCT method $\left(0.3 \mathrm{~km} \mathrm{~s}^{-1}\right)$.

In Figure 4 (left), we present the longitudinal magnetic field $B_{z}$ of NOAA AR 10365 overplotted with the 3D DAVE4VM velocities associated with the occurrence of 2003 May 28 X3.6 flare. The horizontal flows are up to $6 \mathrm{~km} \mathrm{~s}^{-1}$, and the contour levels for vertical flows are $0.1,0.2$, and $0.3 \mathrm{~km} \mathrm{~s}^{-1}$. Enhancement of the $3 \mathrm{D}$ flow fields (i.e., diverging and vertical flows) seem to be spatially correlated with the flare around the same segment of the PIL. Note that these are mainly to test the implementation of the DAVE4VM technique on the ground-based magnetograph data. The observed flow signal during the flare may not be true as the magnetic field measurement can be seriously affected by flare emissions. Moreover, seeing variation can also contribute to about $1 \mathrm{~km} \mathrm{~s}^{-1}$ noise in the velocity measurement.

Figure 4 (right) shows $B_{z}$ of NOAA AR 11057 on 2010 March 29 overplotted with the 3D DAVE4VM velocities (same as above but the contour levels are 0.01 and 

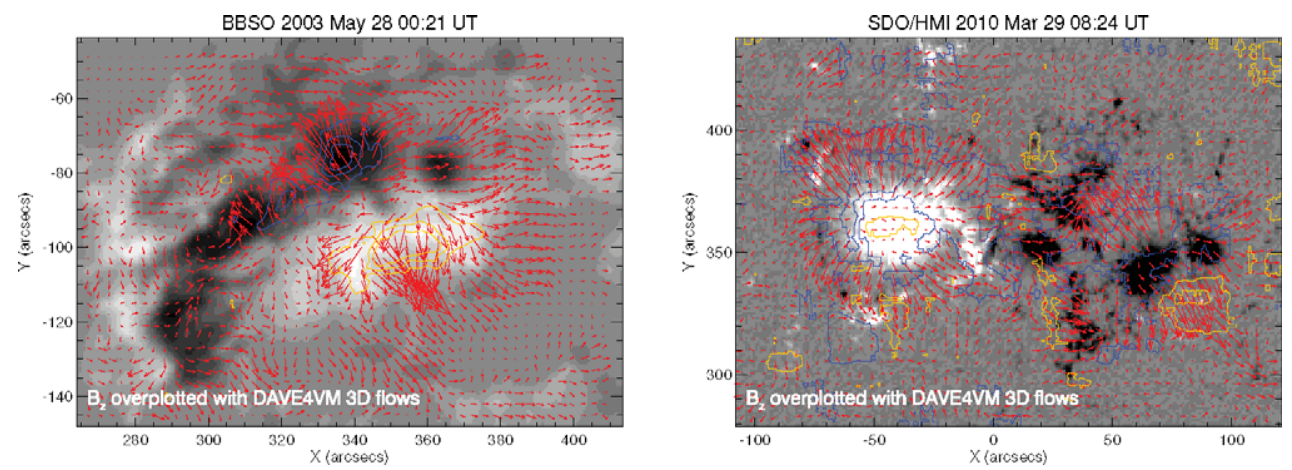

Figure 4. 3D flow fields of NOAA AR 10365 (left) and NOAA AR 11057 (right). Red arrows denote horizontal flows, and blue and yellow contours represent upflows and downflows, respectively.

$0.03 \mathrm{~km} \mathrm{~s}^{-1}$ ). The horizontal flow field exhibits super-penumbral flows corresponding to moving magnetic features, the magnitude of which is about $0.3 \mathrm{~km} \mathrm{~s}^{-1}$.

\section{Summary}

Using Hinode G-band data spanning the X3.4 flare occurred in NOAA AR 10930 on 2006 December 13, we found a sudden change of shearing motion to unshearing motion between the two spots with opposite polarities after the flare. We obtained the flow fields with both DAVE and FLCT methods, and computed the evolution of shear flow around the flaring PIL. A gradual decrease of average velocity of the shear flow is found to be associated with the flare, with a magnitude of $0.2 \mathrm{~km} \mathrm{~s}^{-1}$.

We obtained the 3D velocity field of NOAA AR 10365 on May 28, 2003 using DAVE4VM. Enhancement of 3D flow fields seem to be associated with the occurrence of an X3.6 flare. Note that these are mainly to test the implementation of the DAVE4VM technique on the ground-based magnetograph data. The observed flow signal during the flare may be affected by flare emissions as well as seeing variation.

DAVE4VM 3D velocity field of NOAA AR 11057 on 2010 March 29 was also derived using HMI vector magnetogram. This active region shows a horizontal super-penumbral flows corresponding to moving magnetic features, the magnitude of which is about 0.3 $\mathrm{km} \mathrm{s}^{-1}$. The vertical flow is smaller than $0.1 \mathrm{~km} \mathrm{~s}^{-1}$.

\section{Acknowledgement}

We thank Dr. Peter Schuck for providing the DAVE and DAVE4VM codes. We thank Dr. Yang Liu and the HMI team for processing and providing the excellent HMI magnetograms. The presentation of this paper in the IAU Symposium 273 was made possible due to partial support from the National Science Foundation grants ATM 0548260 and AST 0968672, and NASA Living With a Star grant 09-LWSTRT09-0039.

\section{References}

Kosugi, T., Matsuzaki, K., Sakao, T., Shimizu, T., \& Sone, Y., et al. 2007, Solar Phys., 243, 3 Liu, C., Deng, N., Liu, Y., Falconer, D., Goode, P. R., Denker, C., \& Wang, H. 2005, Astrophys. J., 622,722

Schuck, P. W 2006, Astrophys. J., 646, 1358 
Schuck, P. W 2008, Astrophys. J., 683, 1134

Tan, C., Chen, P. F., Abramenko,V., \& Wang, H. 2009, Astrophys. J., 690, 1820

Tsuneta, S., Ichimoto, K., Katsukawa, Y., Nagata, S., Otsubo, M., Shimizu, T., Suematsu, Y., Nakagiri, M., Noguchi, M., \& Tarbell, T., et al. 2008, Solar Phys., 249, 167

Wang, H. 2006, Astrophys. J., 649, 490

Welsch, B. T., Fisher, G. H., \& Abbett, W. P. 2004, Astrophys. J., 620, 1148 